\title{
Is All of Cancer Genetic?
}

Dawna M. Gilchrist MD

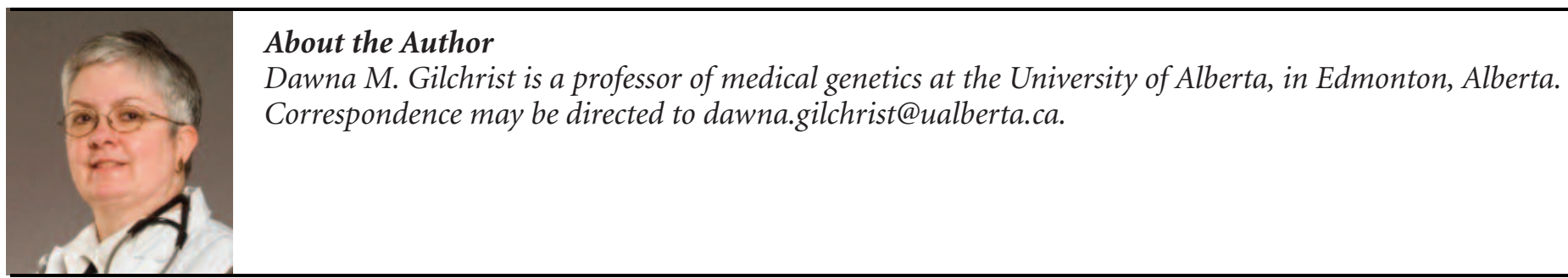

\section{Summary}

There is a genetic component, small or large, to every cancer. Understanding the location of the abnormality informs whether the cancer is heritable (germline) or not (somatic).

\section{Résumé}

Tout cancer comporte une composante génétique plus ou moins importante. En analysant où se situe l'anomalie, on peut obtenir des données sur le caractère héréditaire du cancer (cellules germinales ou somatiques).

$\mathrm{O}$ ver the past 20 years, an appreciation for the genetic contribution to cancer has been impressed not only on the medical profession but also on the public. When famous celebrities (such as Angelina Jolie) announce that they are affected by a hereditary cancer genetic syndrome, patient requests for referrals for that syndrome go up exponentially. Yet very few cancers are actually due to a single detectable genetic cause.

\section{Multifactorial Cancer}

Overall, 90 to $95 \%$ of any type of cancer in the population is multifactorial. That is, the cancer is due to small additive factors from genetic, environmental, and personal domains, adding up to "enough" (Figure 1). The genetic factors come from our complete genetic complement where some genes will predispose to cancer, some will protect from cancer, and most will be neutral. The environment consists of all the food and water we consume over our lives, contaminants in the air or the soil, and any toxins we might come into contact with (herbicides, pesticides, household cleaners, work-related inhalants, etc.). The personal factors include age, good or bad health, good or bad habits, and even good or bad luck.

Multifactorial cancer is too complex in its origins to be currently amenable to any diagnostic test - genetic or otherwise.

\section{“Single" Factors}

A minority of cancers are due to "single" factors (Figure 2). The multiple factors of genetic, environment, and personal domains still exist but pale in comparison to an identifiable single driver for cancer.

Some single gene mutations cause cancer genetic syndromes and are capable of being passed from generation to generation. Alternatively, cancer might be due to an identifiable

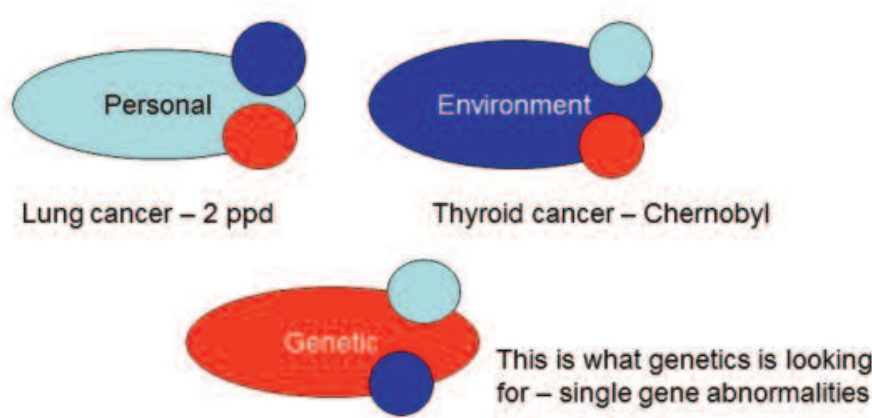

Figure 2. Unequal components.

Figure 1. Of cancers, 90 to $95 \%$ are multifactorial.

\section{Multiple, small.}

\pm defined environmental

factors

Multiple, small \pm defined personal factor

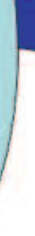

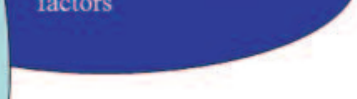


environmental cause - thyroid cancer in a person who lives near Chernobyl or an increased risk of breast cancer after mantle radiation for lymphoma. Or cancer may be due to an infection - human papillomavirus (cervical) or primary hepatocellular carcinoma. Of the personal factors, smoking is the archetypical habit that increases the risk for many types of cancer.

\section{Genetic Syndromes}

A small portion of any type of cancer is due to a single gene mutation that causes a recognizable genetic syndrome. There are currently dozens of known cancer syndromes associated with single gene mutations. In many of these syndromes, the gene(s) responsible have been found; in others, not.

In the vast majority of these syndromes, it is the mutated form of the associated gene that causes the cancer - not its wildtype, normal counterpart. Therefore, when people ask if they have the $B R C A$ gene, what they really mean is, "Do I have a mutated BRCA1 or BRCA2 gene?"

\section{Germline versus Somatic}

In order for a genetic abnormality to be transmissible from generation to generation, it must be in the germline, such as in the egg or sperm. In general, a germline mutation is present in all cells of the body, including any resulting tumours.

Tumours, by their nature, carry many genetic abnormalities. Most of these are limited just to the tumour, such as $k$-ras, Her2-neu, or the translocation that activates an oncogene to cause leukemia (the Philadelphia chromosome). Genetic aberrations that are limited to the tumour tissue only are called somatic. A somatic mutation is not heritable.

Some genes can be found in the mutated form in either germline or somatic situations. For example, germline MLH1 mutations are associated with Lynch syndrome. However, many colorectal cancers display MLH1 deficiency that is somatic only. The deficiency is due to hypermethylation of the MLH1 gene only in the tumour tissue, resulting in cancer. Therefore, a loss of the MLH1 protein in colon cancer is often not indicative of a hereditary condition. Similarly, somatic TP53 mutations are common in many tumours. Rarely, TP53 can be mutated in the germline, causing the syndrome of Li-Fraumeni.

The importance of finding a germline mutation is that it can define further risks in other tissues in the affected individual and also indicate an increased cancer risk for close relatives. For example, a germline BRCA1 mutation confers an increased risk to a woman for both breast and ovarian cancers. As well, this same woman can pass on her germline mutation, at a risk of $50 \%$, in any reproductive event.
The importance of finding a somatic mutation is that this information may help guide therapy. For example, the presence of Her-2-neu allows treatment of breast cancer with the drug trastuzumab (Herceptin).

\section{Basic Facts}

Few people appreciate that cancer eventually affects 1 in 3 in the population. Many of these cancers will be minor skin cancers, but cancer is common. If your family is large and/or people live to old age, there will be cancer. There are only so many ways to die.

Hereditary cancer is equal opportunity in transmission from parent to offspring. For example, BRCA mutations can be inherited from either the maternal or paternal line. Most of the known cancer syndromes are autosomal dominant, and gene mutations do not care whether they are carried by a mother or father (although the risk of expression might be significantly different). The carrier of an autosomal dominant mutation has a 50:50 chance of passing on that mutation in any reproductive event - it's a heads or tails situation.

Early-onset cancer with a family history of the same (or genetically related) cancer is very suggestive of a genetic cancer syndrome. However, young individuals with early-onset cancer but without a significant family history are unlikely to have a germline mutation. Bad luck is equal opportunity at any age.

\section{What Does a Medical Genetics Clinic Look For?}

Medical genetics clinics search for a number of factors:

1. Numbers of affected family members with specific types of cancer. We want to know about the full size of the family, including offspring, siblings, aunts, uncles, cousins, and grandparents. Is the population prevalence of cancer exceeded?

2. The age of onset of cancer in these individuals.

3. Relationships of affected individuals compatible with autosomal dominant inheritance, such as a parent-to-child transmission.

4. A combination of cancers known to relate genetically. It is the breast ovarian cancer syndrome, not the breast uterine or the breast cervix cancer syndrome.

5. Records. A woman who is reported to have ovarian cancer at age 23, who then goes on to have children, more likely had cervical cancer or something benign. A person who is reported to have had breast and ovarian cancers might just have had breast cancer that metastasized to the ovaries.

6. An informative family member to test. Generally, this is someone who has a type of cancer associated with the 
suspected syndrome. We do not (yet) have a comprehensive "cancer genetics test." Unless one has a test that offers 100\% detection for the genetic cause, then initiating testing on an unaffected individual can never rule out excess risk. Only if a causative mutation is identified in an informative affected family member can predictive testing be reliably conducted for an unaffected at-risk family member.

\section{Why Bother with Genetic Counselling?}

A trained cancer genetics counsellor can assess a patient's risk based on an appropriately explored family pedigree, a review of relevant pathology records, and any other information that may be pertinent. Discussion includes other medical risks associated with a cancer syndrome, options for screening, medical or surgical risk reduction, genetic testing, and risks for close relatives. Ancillary topics such as implications for insurance, emigration, and lifestyle choices are also addressed.

Patients often come to medical genetics clinics with unachievable expectations created by TV programs such as CSI or cancer myths they have acquired from family lore. These misconceptions can be difficult and time consuming to dislodge.

\section{Genetic Testing}

If a genetic syndrome is defined and the patient meets clinical criteria, a clinical test will be available only if the causative gene(s) has been localized and cloned. Mutations in BRCA1 and $B R C A 2$ account for only about $50 \%$ of the incidences of hereditary breast ovarian cancer syndrome and about $20 \%$ of hereditary breast cancers. The respective remainders are due to genes that have not yet been elucidated or for which funded testing is not readily available.

Availability of cancer genetics testing varies by province. Some is done "in house" while other testing is farmed out to provincial molecular diagnostic laboratories or laboratories in the United States or Europe. Different provinces have various policies for the approval of funding for out-of-province testing. Genetic testing for anything must be done in the province where the individual holds health care. Payment for genetic testing is not automatically transferrable from one province's health care system to another. Much in the way of cancer genetics testing is restricted to ordering by Cancer Genetics Clinics. In general, cancer genetic testing is not inexpensive; nor is it quick.

\section{Summary}

Cancer always has a genetic component, but most of the genetic component is part of a multifactorial etiology, and detectable genetic abnormalities are localized to the tumour only. Singlegene abnormalities that cause heritable cancer syndromes are relatively rare, and the family history is still the best tool in their detection.

\section{Recommended Resources}

Foulkes W. Inherited susceptibility to common cancers. N Engl J Med 2008;359;20:2143-53.

GeneReviews: www.ncbi.nlm.nih.gov/gtr/.

McDermott U, Downing JR, Stratton MR. Genomics and the continuum of cancer care. N Engl J Med 2011;364;4:340-50.

National Comprehensive Cancer Network: www.nccn.org.

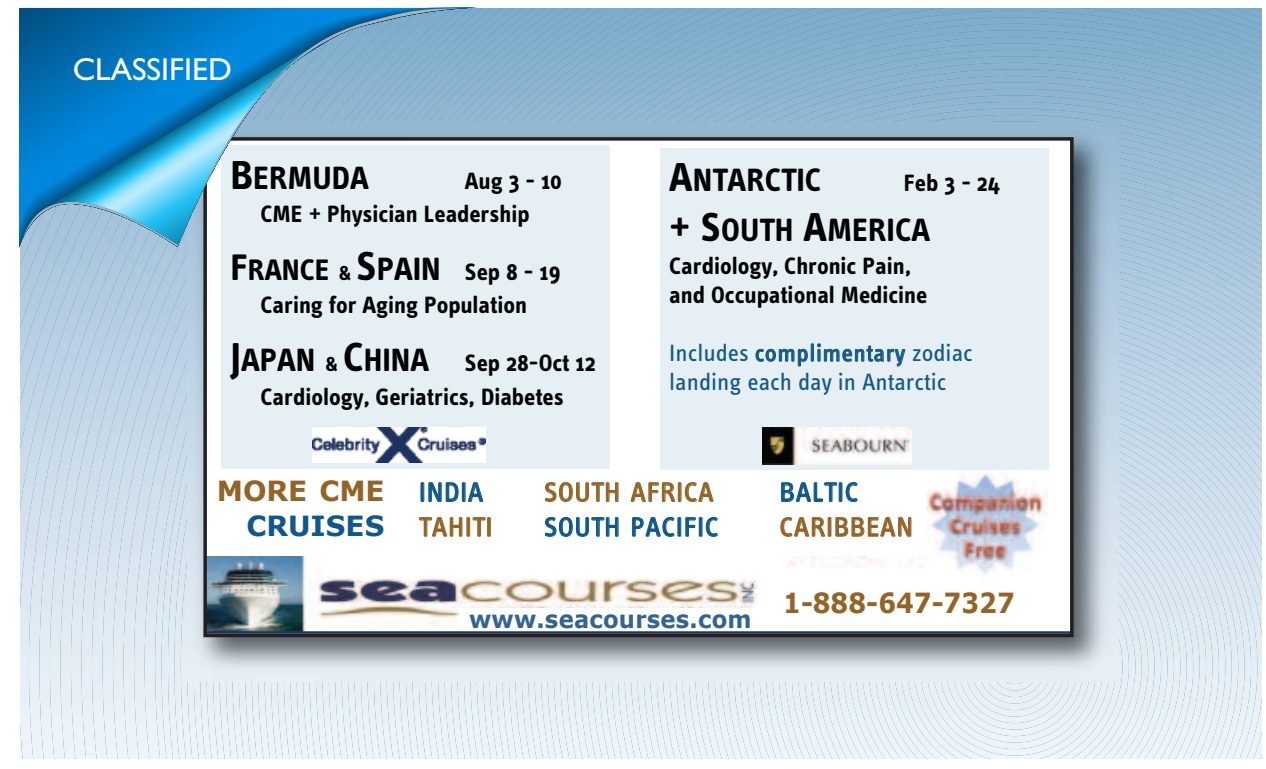

